\title{
CARACTERÍSTICAS SOCIODEMOGRÁFICAS Y DE SALUD DE LOS ADULTOS MAYORES EN SITUACIÓN DE CALLE EN LIMA, PERÚ
}

\author{
Marlene Moquillaza-Risco ${ }^{1, a}$, Elsa León ${ }^{1, a}$, Mario Dongo ${ }^{1, b}$, César V. Munayco ${ }^{1, c}$
}

\begin{abstract}
RESUMEN
Objetivos. Describir las características sociodemográficas y de salud de las personas adultas mayores en situación de calle (PAMSC). Materiales y métodos. Estudio de tipo transversal, se identificaron variables sociodemográficas y de salud de las PAMSC al momento del ingreso al Programa Nacional Vida Digna (PNVD) del Ministerio de la Mujer y Poblaciones Vulnerables del Perú. Se realizó un análisis descriptivo de las variables sociodemográficas y de las condiciones de salud, además se determinó la probabilidad de dependencia funcional según edad y estratificada por sexo y estado mental, usando un modelo de regresión logística. Resultados. Las PAMSC fueron, en su mayoría, hombres, solteros y con grado de instrucción primaria o menor. El 66,2\% de las PAMSC presentaron algún tipo de enfermedad crónica y 27,8 algún tipo de enfermedad mental. El 50\% presentaron algún grado de dependencia funcional, y casi el $70 \%$ presentó algún grado de deterioro cognitivo. La probabilidad de dependencia funcional parcial se incrementó con la edad, siendo más alta en mujeres, además, se incrementó a mayor grado de deterioro cognitivo. Conclusiones. Las PAMSC son una población altamente vulnerable no solo por el hecho de vivir a la intemperie, con necesidades básicas insatisfechas, sino también por su alta frecuencia de enfermedades crónicas y mentales, además de su alto grado de dependencia funcional y deterioro mental, que hace difícil que puedan valerse por sí mismas y requieren de cuidados especiales para superar esta situación de abandono.
\end{abstract}

Palabras clave: Adulto mayor; Persona sin hogar; Envejecimiento; Deterioro cognitivo leve (fuente: DeCS BIREME).

\section{SOCIO-DEMOGRAPHICS CHARACTERISTICS AND HEALTH CONDITIONS OF OLDER HOMELESS PERSONS OF LIMA, PERU}

\begin{abstract}
Objectives. Determine the socio-demographics characteristics and health conditions of older homeless persons at the time of enrollment into the National Program "Vida Digna" and the probability of functional dependency by age, and stratified by gender and cognitive impairment. Materials ande methods. We performed a cross sectional study, reviewing all registration forms of the program in order to identify socio-demographic variables and health conditions of older homeless persons at the time of enrollment in the program. We did a descriptive analysis of the socio-demographic variables and we also determined the frequency of health conditions. Furthermore, we determined the probability of functional dependency by age, and stratified by gender and cognitive impairment through a logistic regression model. Results. The older homeless persons at the time of enrollment in the program were mostly single men, with a primary education or no education. The study subjects had a high frequency of chronic and mental diseases. $50 \%$ of them had certain level of functional impairment and roughly $70 \%$ had a certain level of cognitive impairment. The probability of functional dependency increased by age, and it was higher in women than in men. This probability increased according to the level of cognitive impairment. Conclusions. This study shows that older homeless persons are a vulnerable population not only because they live outdoors but also because they a have also for the high prevalence of chronic and mental diseases. These diseases prevent the homeless persons from living by themselves special care to overcome their situations.
\end{abstract}

Key words: Elderly; Homeless person; Aging; Mild cognitive impairment (source: MeSH NLM).

\section{INTRODUCCIÓN}

El envejecimiento es un proceso fisiológico natural e irreversible, pero también es un triunfo de las políticas de salud que han permitido que las personas tengan una vida más larga (1). Este hecho genera nuevos retos para el Estado peruano que debe proveer los servicios necesarios para la protección y atención de las personas adultas mayores para que vivan más años con calidad de vida. En este sentido, el Estado debe generar políticas públicas para garantizar que las personas que envejecen tengan una vida placentera, activa y con independencia en familia y en comunidad ${ }^{(2,3)}$.

\footnotetext{
Programa Nacional Vida Digna (PNVD), Ministerio de la Mujer y Poblaciones Vulnerables. Lima, Perú.

a Licenciada en Asistencia Social; b estudiante de Ingeniería Ambiental; ${ }^{c}$ médico, magíster en Epidemiología y Salud Pública

Recibido: 10-04-15 Aprobado: 23-07-15
} 
Desafortunadamente, existe un grupo de personas adultas mayores invisibles a la sociedad, que viven en situación de alta vulnerabilidad social y con riesgo permanente, que han quedado excluidos y desvinculados de sus entornos familiares, y muchas veces despreciados por su entorno social ${ }^{(2)}$. Este grupo son las personas adultas mayores en situación de calle (PAMSC), que no solo son pobres extremos sino que viven en una situación casi inhumana ${ }^{(4-6)}$, sin hogar ni servicios básicos, sin satisfacción de necesidades básicas, sin acceso a los servicios de salud, sin acceso a recreación, desvinculados y olvidados por sus familias y por el estado ${ }^{(7-9)}$

Las personas en situación de calle tienen, además, del deterioro propio de la edad, un inaceptable alto riesgo de enfermedades prevenibles (neumonía, influenza), adicciones (drogadicción, alcoholismo), victimización (violaciones sexuales), morbilidad progresiva (enfermedades crónicas, cáncer, etc.) y un expectativa de vida por debajo del promedio nacional ${ }^{(4,10)}$.

Actualmente, existe sólida evidencia que demuestra el éxito de los programas sociales dirigidos a las personas en situación de calle ${ }^{(11-13)}$. Estas evidencias señalan que el alojamiento y manejo adecuado de las personas en situaciones de calle, conduce a una reinserción social, reducción de visitas a emergencias y hospitalizaciones debido a reducción de complicaciones, control de adiciones y, sobre todo, incremento de la expectativa de vida y mejora de la calidad de vida ${ }^{(14-20)}$

Uno de los factores decisivos para la reinserción social/familiar de las personas en situación de calle, especialmente las personas adultas mayores, es su grado de dependencia funcional y cognitiva, es decir, su capacidad de ejecutar las actividades básicas de la vida diaria (ABVD) tales como vestirse, comer o asearse, y las que se engloban en el concepto de actividades instrumentales de la vida diaria (AIVD), tales como comprar o preparar comida ${ }^{(21)}$.

En el 2012, el Gobierno peruano, en su afán de abordar este problema, creó el programa Nacional Vida Digna (PNVD) con la finalidad de restituir y proteger los derechos de las PAM/SC. EI PNVD ofrece la restitución de su derecho a la identidad, acceso al Seguro Integral de Salud (SIS), alojamiento permanente o nocturno, alimentación, vestido y cuidados propios de la edad (22). Actualmente el PNVD atiende en sus servicios de acogida a 457 PAM/SC en las ciudades de Lima, Arequipa, Huaraz y Huánuco.

En el Perú, a pesar de los esfuerzos del gobierno por abordar este problema, poco se sabe sobre este grupo de personas, porque es una población móvil y difícil de abordar. Por este motivo, con el fin de generar evidencias que ayuden a implementar mejores políticas públicas orientadas a prevenir y superar este problema, el objetivo de este estudio fue identificar y describir las características sociodemográficas y de salud basales de las PAMSC a través del estudio de los beneficiarios del PNVD, al momento del ingreso a dicho programa. Además, se determinó la probabilidad de dependencia funcional por edad, y estratificada por sexo y deterioro cognitivo.

\section{MATERIALES Y METÓDOS}

\section{DISEÑO DEL ESTUDIO}

Se realizó un estudio transversal, para lo cual se revisaron los archivos del PNVD, identificándose las siguientes fuentes de datos: las fichas iniciales de contacto, la ficha de evaluación económica, la ficha de salud, el informe social y el de psicología de cada beneficiario del PNVD.

\section{ÁREA Y POBLACIÓN DE ESTUDIO}

Este estudio se realizó en personas adultas mayores de 60 a más años, en situación de calle, que ingresaron al PNVD entre el 2012 y junio de 2014 en todas la sedes del programa (Lima, Perú), y que se encuentran acogidos en algún Centro de Atención Residencial para Personas Adultas Mayores (CARPAM) o en alguna hospedería, a nivel nacional. Los CARPAM ofrecen alojamiento permanente, cuidados de enfermería, vestimenta, alimentación completa y atenciones en salud. En cambio, las hospederías ofrecen alojamiento nocturno, vestimenta, alimentación y atenciones en salud. En ambos servicios se trabaja complementariamente talleres y actividades sociorecreativas que propician el fortalecimiento de capacidades para optimizar el proceso de envejecimiento.

\section{RECOLECCIÓN DE DATOS}

Entre junio y julio de 2014, se revisaron los archivos del PNVD con la finalidad de identificar las fuentes de información, para determinar la línea de base del programa respecto a las características de los beneficiarios. Se identificaron cinco fuentes de información que a continuación se describen.

La ficha inicial de contacto, es llenada por los facilitadores del PNVD que se encargan de focalizar a las personas adultas mayores en las calles con la finalidad de motivarlos a dejar la situación de calle e invitarlos al PNVD. Esta ficha recoge información demográfica (edad, sexo, grado de instrucción, estado civil, lugar de nacimiento), si es que tiene el documento nacional de identidad, dónde duerme, si tiene familiares, si tiene algún tipo de seguro, o si sufre de alguna enfermedad. 
Esta es la ficha que sirve de tamizaje para identificar a los potenciales beneficiarios del PNVD. Además, contiene datos relacionados a la salud del beneficiario, que son autorreportados por el beneficiario.

La ficha de evaluación económica evalúa qué tipo de trabajo realiza, y si está afiliado a algún programa social. La ficha de salud hace una evaluación general de la salud, y el estado de dependencia/independencia del beneficiario, a través de índice de valoración funcional de KATZ. Este test evalúa el grado de dependencia/ independencia de las personas utilizando seis funciones básicas: baño (esponja, ducha o bañera), vestido, uso del retrete, movilidad, continencia y alimentación ${ }^{(23)}$. En esta escala cada actividad se categorizaba en tres niveles: independencia, dependencia parcial y dependencia total.

De acuerdo al índice de valoración funcional de KATZ, se considera independiente a una persona que no precisa ayuda o utiliza ayuda solo para un componente de la actividad, y dependiente total a aquella que necesita ayuda de otra persona, incluyendo supervisión o guía, para todos los componentes de la actividad; finalmente dependiente parcial es aquella persona que "requiere de asistencia" para más de un componente, pero que puede realizar otros componentes de la actividad sin ayuda o supervisión ${ }^{(24)}$

La evaluación social determina si realmente esta persona cumple con el perfil establecido de PAM/SC, excluido socialmente o no (requisito indispensable para ingresar al PNVD), la ficha de psicología evalúa, entre otras cosas, el estado cognitivo con el test de Pfeiffer, y el estado afectivo con el test de Yesavage. El Pfeiffer valora el deterioro cognitivo a través de cuestionario de 10 ítems, sobre un pequeño número de funciones relativamente básicas (memoria de corto y largo plazo, atención, orientación, información sobre hechos cotidianos, o capacidad matemática) ${ }^{(25)}$.

Esta escala categoriza el desarrollo cognitivo en: normal, deterioro cognitivo leve, deterioro cognitivo moderado (patológico), deterioro cognitivo severo.

El test de Yesave se aplica a personas adultas mayores y se enfoca a evaluar el estado de ánimo, es decir, depresión geriátrica ${ }^{(26)}$. Es importante tener en cuenta que todos los resultados de los test descritos fueron recogidos de las fichas mencionadas anteriormente.

\section{ANÁLISIS DE DATOS}

Los datos de las diferentes fichas fueron ingresados a una base de datos en Microsoft Excel y convertidos al formato "csv" para ser analizados con el paquete "epicalc" del lenguaje de programación R (27). Para proteger la identidad de los beneficiarios del PNVD se encriptó los nombres y apellidos, y se les asignó un código correlativo.

Se realizó un análisis descriptivo en base a distribución de frecuencias de las principales variables sociodemográficas como sexo, grado de instrucción, estado civil, etc. Además, se calculó la mediana, y los valores mínimo y máximo de la variable edad. Se determinaron las frecuencias relativas de las enfermedades físicas y mentales de los sujetos de estudio, así como el estado de dependencia y el estado mental.

Se determinó la probabilidad de dependencia parcial (no hubo beneficiarios con dependencia total) de acuerdo con la edad, tanto para hombres y mujeres, a través de un modelo de regresión logística. Finalmente, se determinó la probabilidad de dependencia parcial de acuerdo con la edad y el grado de deterioro cognitivo.

\section{CONSIDERACIONES ÉTICAS}

Este estudio tuvo la aprobación de la Dirección Ejecutiva del Programa Nacional Vida Digna para la revisión de los archivos de dicha Dirección. Para asegurar la confidencialidad de los beneficiarios no se registró ningún dato que pudiera identificarlos y se usaron códigos de identificación.

\section{RESULTADOS}

Se recolectó datos de 348 beneficiarios del PNVD, se excluyó a 46 beneficiarios por no contar con el dato de edad o ser incierto, o si la edad fue menor de 60 (uno de los criterios para ingresar al PNVD es ser mayor de 60 años) o mayor de 100 años (probable error en el registro).

La mediana de edad de los beneficiarios al momento del ingreso al PNVD fue de 72 años, y en su mayoría $(82,8 \%)$ fueron varones. Con respecto al grado de instrucción, el $37,8 \%$ tiene primaria completa o incompleta, y el $10,9 \%$ no tiene instrucción. Es importante resaltar que el $8 \%$ tiene nivel universitario. En su mayoría son solteros $(69,6 \%)$, y solo el $15,8 \%$ fueron casados. Existen muchas formas como la PAM/ SC es identificada y referida al PNVD, en nuestro caso, la mayoría de la PAM/SC fueron identificadas o referidas a través de los facilitadores del programa $(65,6 \%)$, o por otras instituciones como la Policía Nacional $(7,4 \%)$. Solo $5 \%$ fueron por solicitud propia de la PAMSC (Tabla 1). 
Tabla 1. Características sociodemográficas de los beneficiarios del Programa Nacional Vida Digna (PNVD)

\begin{tabular}{|c|c|c|}
\hline Características & $\mathbf{n}$ & $(\%)$ \\
\hline Edad al ingreso (años)* $(n=302)$ & 72 & $(60-96)$ \\
\hline \multicolumn{3}{|l|}{$\operatorname{Sexo}(n=302)$} \\
\hline Masculino & 250 & $(82,8)$ \\
\hline Femenino & 52 & $(17,2)$ \\
\hline \multicolumn{3}{|l|}{ Grado de instrucción $(n=238)$} \\
\hline Sin instrucción & 26 & $(10,9)$ \\
\hline Primaria incompleta & 27 & $(11,3)$ \\
\hline Primaria completa & 63 & $(26,5)$ \\
\hline Secundaria incompleta & 16 & $(6,7)$ \\
\hline Secundaria completa & 83 & $(34,9)$ \\
\hline Superior técnica & 4 & $(1,7)$ \\
\hline Superior universitaria & 19 & $(8,0)$ \\
\hline \multicolumn{3}{|l|}{ Estado civil $(n=240)$} \\
\hline Soltero & 167 & $(69,6)$ \\
\hline Casado & 38 & $(15,8)$ \\
\hline Viudo & 26 & $(10,8)$ \\
\hline Divorciado & 2 & $(0,9)$ \\
\hline Separado & 7 & $(2,9)$ \\
\hline \multicolumn{3}{|c|}{ Medio de identificación de la PAMSC ( $n=299)$} \\
\hline Facilitador & 196 & $(65,6)$ \\
\hline Policía Nacional del Perú & 22 & $(7,4)$ \\
\hline Sociedad civil & 21 & $(7,0)$ \\
\hline Vía telefónica por cualquier ciudadano & 17 & $(5,7)$ \\
\hline Fiscalía & 11 & $(3,7)$ \\
\hline Solicitud propia & 15 & $(5,0)$ \\
\hline Hospital & 8 & $(2,7)$ \\
\hline Municipalidad & 4 & $(1,3)$ \\
\hline Beneficencia & 4 & $(1,3)$ \\
\hline Defensoría del Pueblo & 1 & $(0,3)$ \\
\hline \multicolumn{3}{|l|}{ Condición actual de la PAMSC (n=300) } \\
\hline Alojado en CARPAM & 143 & $(47,7)$ \\
\hline Servicio de hospedería & 77 & $(25,7)$ \\
\hline Egresado & 57 & $(19,0)$ \\
\hline Fallecido** & 23 & $(7,6)$ \\
\hline
\end{tabular}

* Mediana (mínimo-máximo), "entre el inicio del programa y junio 2014. PAMSC: persona adulta mayor en situación de calle

Hasta junio de 2014 , el $47,7 \%$ de los beneficiarios del PNVD se encontraban alojados en un CARPAM, o usaban el servicio de hospedería (25,7\%). El $19 \%$ y el $7 \%$ había egresado del PNVD o fallecido, respectivamente (Tabla 1).

Con respecto a la red familiar de la PAMSC, solo 29 $(9,6 \%)$ de ellos tiene algún familiar cercano. Entre los familiares cercanos, 15 (5,0\%) son hijo(a)/hijos(as), 9 $(3,0 \%)$ son hermanos, y $3(1,0 \%)$ sobrinos (datos no incluido en la Tabla 1).

EI $66,2 \%$ de las PAMSC presentaron alguna enfermedad crónica al momento del ingreso al PNVD, como hipertensión arterial $(14,2 \%)$, artritis/artrosis $(7,6 \%)$, y diabetes mellitus (5,6\%), entre otras. Además, algunos tuvieron antecedentes de enfermedad infecciosa como sífilis (5\%), o tuberculosis (2,9\%) (Tabla 2). Es importante destacar que muchas de las PAMSC sufren de varias enfermedades a la vez. A esto se le suma que algunas PAMSC tienes problemas de alcohol y uso de drogas como marihuana o pasta básica de cocaína. Por otro lado, el $48,9 \%$ de las PAMSC presentaron dependencia funcional parcial al momento de su ingreso al PNVD.

Las enfermedades mentales también fueron prevalentes en este grupo de personas vulnerables, porque el $27,8 \%$ presentó alguna enfermedad mental al momento del ingreso al PNVD, entre las que destacan demencia senil $(10,3 \%)$, algún tipo de esquizofrenia $(6 \%)$ y enfermedad de Parkinson (3\%). Finalmente, el $23,2 \%$ y $12,5 \%$ de las PAMSC presentaron deterioro cognitivo moderado

Tabla 2. Salud física y mental de los beneficiarios del Programa Nacional Vida Digna (PNVD)

\begin{tabular}{|c|c|c|}
\hline Salud física y mental & $\mathbf{n}$ & $(\%)$ \\
\hline \multicolumn{3}{|l|}{$\begin{array}{l}\text { Enfermedades crónicas reportadas al momento } \\
\text { del ingreso }(n=302)\end{array}$} \\
\hline Sí & 200 & $(66,2)$ \\
\hline \multicolumn{3}{|l|}{ Prevalencia de enfermedades crónicas ( $n=302$ ) } \\
\hline Hipertensión arterial & 43 & $(14,2)$ \\
\hline Artritis/Artrosis & 23 & $(7,6)$ \\
\hline Diabetes mellitus & 17 & $(5,6)$ \\
\hline Desnutrición crónica & 14 & $(4,6)$ \\
\hline Secuela de enfermedad cerebro vascular & 14 & $(4,6)$ \\
\hline Insuficiencia renal crónica & 2 & $(0,7)$ \\
\hline \multicolumn{3}{|l|}{ Prevalencia de enfermedades infecciosas (302) } \\
\hline Sífilis (VDRL positivo) & 15 & $(5,0)$ \\
\hline Antecedente de tuberculosis & 9 & $(2,9)$ \\
\hline Hepatitis viral B/C & 5 & $(1,6)$ \\
\hline VIH/SIDA & 2 & $(1,0)$ \\
\hline \multicolumn{3}{|c|}{ Prevalencia de uso de drogas legales e ilegales $(n=302)$} \\
\hline Consumo de alcohol & 48 & $(19,1)$ \\
\hline Consumo de marihuana/pasta básica de cocaína & 9 & $(3,0)$ \\
\hline \multicolumn{3}{|l|}{ Valoración funcional (Test de KATZ) (n=280) } \\
\hline Independiente & 143 & $(51,1)$ \\
\hline Dependiente parcial & 137 & $(48,9)$ \\
\hline \multicolumn{3}{|c|}{$\begin{array}{l}\text { Enfermedad mental reportada al momento del ingreso al } \\
\text { PNVD }(n=307)\end{array}$} \\
\hline Sí & 84 & $(27,8)$ \\
\hline \multicolumn{3}{|l|}{ Prevalencia de enfermedad mental $(n=302)$} \\
\hline Demencia senil & 31 & $(10,3)$ \\
\hline Esquizofrenia & 18 & $(6,0)$ \\
\hline Enfermedad de Parkinson & 9 & $(3,0)$ \\
\hline Trastornos psicóticos & 4 & $(1,3)$ \\
\hline Retardo mental & 2 & $(0,7)$ \\
\hline Trastorno bipolar & 2 & $(0,7)$ \\
\hline \multicolumn{3}{|l|}{ Valoración del estado mental (Test de Pfizzer) $(n=280)$} \\
\hline Normal & 94 & $(33,6)$ \\
\hline Deterioro cognitivo leve & 86 & $(30,7)$ \\
\hline Deterioro cognitivo moderado & 65 & $(23,2)$ \\
\hline Deterioro cognitivo severo & 35 & $(12,5)$ \\
\hline
\end{tabular}

PAMSC: persona adulta mayor en situación de calle 


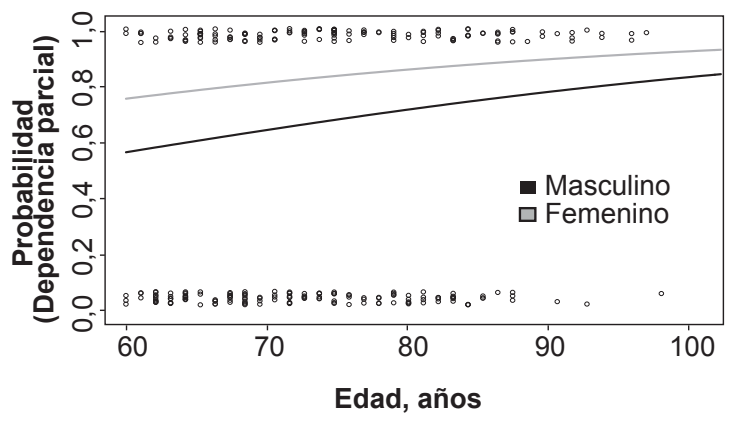

Figura 1. Probabilidad de dependencia parcial según edad estratificada por sexo del beneficiario del Programa Nacional Vida Digna

y severo, respectivamente, al momento del ingreso al PNVD (Tabla 2).

La valoración sociofamiliar de las PAMSC, mostró que el $100 \%$ se encontraban en riesgo social, por no contar con apoyo familiar y una red de soporte social. Además, el test de Yesave arrojó que el 100\% de las PAMSC presentaron algún grado de depresión (datos no incluidos en la Tabla 2).

La probabilidad de dependencia funcional parcial se incrementa con la edad, tanto en hombres como en mujeres. Además, las mujeres presentan una mayor probabilidad de dependencia funcional parcial que los hombres (Figura 1). Por ejemplo, la probabilidad de dependencia parcial se incrementa de 0,75 a los 60 años de edad, hasta casi 0,90 a los 80 años. En cambio, en el hombre esta probabilidad se incrementa de 0,6 a los 60 años de edad, hasta casi 0,70 a los 80 años.

La probabilidad de dependencia funcional parcial se incrementa con la edad en todos los grados de deterioro cognitivo, excepto en el deterioro cognitivo severo, donde en todos las edades, la probabilidad de dependencia funcional parcial es cercana a 1. Por otro lado, la dependencia funcional parcial se incrementa con el grado de deterioro cognitivo (Figura 2). Por ejemplo, en aquellas PAMSC sin deterioro cognitivo,

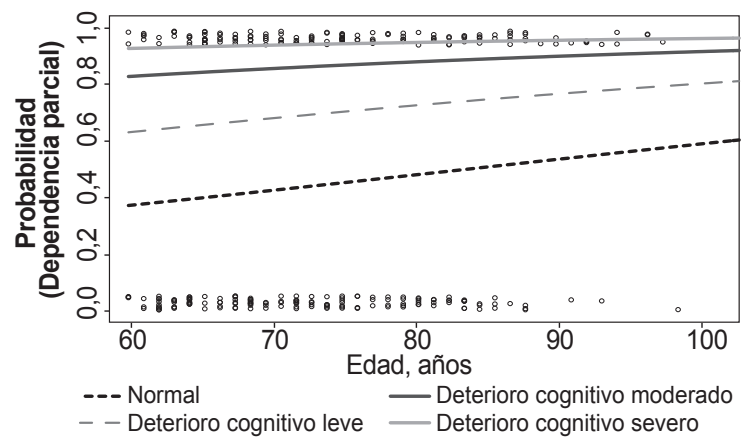

Figura 2. Probabilidad de dependencia parcial según edad estratificada por nivel de deterioro cognitivo del beneficiario del Programa Nacional Vida Digna la probabilidad fluctúa entre 0,4 y 0,5 entre los 60 y 80 años, por el contrario en las PAMSC con deterioro cognitivo leve esta probabilidad fluctúa entre 0,6 y 0,7 entre los 60 y 80 años; y en las PAMSC con deterioro cognitivo moderado esta probabilidad fluctúa entre 0,8 y 0,95 en el mismo rango de edades.

\section{DISCUSIÓN}

Este estudio muestra que las personas PAM/SC al ingreso al PNVD fueron, en su mayoría hombres, solteros y con un grado de instrucción primario o menor. Las PAMSC presentan una alta prevalencia de enfermedades crónicas, entre las que destacan hipertensión arterial, artritis/artrosis y diabetes mellitus. Además, también presentan un alta prevalencia de enfermedades mentales, como demencia senil, esquizofrenia y enfermedad de Parkinson.

Por otro lado, los resultados de este estudio indican que casi el $50 \%$ de los PAM/SC al ingreso al PNVD presentaron dependencia funcional parcial, y casi el $70 \%$ presentó algún grado de deterioro cognitivo. Finalmente, la probabilidad de dependencia funcional parcial se incrementa con la edad, y se observó que esta probabilidad fue mayor en las mujeres que en los hombres, además, esta probabilidad se incrementó grandemente a mayor grado de deterioro cognitivo.

Este estudio presenta las siguientes limitaciones: 1). Debido a que los datos se recolectaron de las fichas de registro del PNVD, los resultados podrían estar subestimados y no ser tan precisos, principalmente cuando nos referimos a la prevalencia de enfermedades crónicas y mentales, ya que estos datos no provienen de un examen médico exhaustivo, sino por el contrario del reporte de la PAM/SC o por la evaluación de los promotores del PNVD, que no son personal de salud. A pesar de este problema, existen enfermedades (como las que hemos mencionado) que son fácilmente reconocibles tanto por el paciente como por el promotor, especialmente en los adultos mayores. 2). Otro problema importante es que las personas con deterioro cognitivo y enfermedades mentales no pueden dar datos precisos. Finalmente, 3) La información sobre la de edad se pudo ver afectada principalmente en las personas que no pueden dar este datos o se niegan a hacerlo, porque es difícil calcular la edad ectoscópicamente en este grupo de personas, porque aparentan mayor edad que la que tienen, por la condiciones en que viven. En muchos casos, la edad verdadera se obtiene después del ingreso de la PAM/SC al PNVD cuando se establece un grado de confianza entre la PAM/SC y el equipo del PNVD o se verifican los datos en el RENIEC. Esto también se observa con los datos relacionados a familiares. 
Una de las metodologías comunes para obtener información e identificar a las personas en situación de calle son los censos o catastros, que son estudios poblacionales en una determinada área geográfica, como una ciudad, así fue en el censo en Medellín Colombia, en Ciudad de México, y el Alto en Bolivia; o incluso en todo el país, como los catastros realizados en Chile en el 2005 y 2011. Estos estudios muestran el mismo patrón demográfico (predominancia de hombres solteros) y educacional (grado de instrucción primario o menor) de las PAMSC, que nuestro estudio ${ }^{(7-9,28)}$. Este patrón probablemente se explique porque los varones solteros tienen menos arraigo familiar y porque el tener un bajo nivel de instrucción les impide encontrar trabajos bien remunerados que puedan cubrir sus necesidades básicas.

Este estudio muestra el importante rol que cumplen los promotores del PNVD para identificar y enrolar a las PAMSC, además del rol de otras instituciones del estado y la sociedad civil (reporte), ya que las PAMSC son difíciles de identificar y abordar por su alta movilidad y comportamiento esquivo a la autoridad, y en muchos casos son los censos o catastros los que ayudan a identificarlos ${ }^{(29,30)}$.

Es indudable que este grupo particular de personas adultas mayores, tiene una alta prevalencia de enfermedades crónicas y mentales, además de enfermedades infecciosas, abuso de alcohol y uso de drogas ilegales que la población adulta mayor $(2,4,31)$. Lamentablemente, debido al autorreporte, en algunos casos estas prevalencias son más bajas de lo esperado en una población adulta mayor, como en el caso de hipertensión arterial ${ }^{(31)}$.

Los censos y catastros que se han realizado en nuestro continente no recogen información de salud por ello no podemos comparar nuestros resultados con otros del continente, por lo que vamos a comparar nuestros resultados con la Encuesta Salud, Bienestar y Envejecimiento (SABE) realizada en varios países de Latinoamérica y el Caribe ${ }^{(31)}$ en personas adultas mayores; por ejemplo, este estudio señala que, en promedio, la prevalencia de enfermedades crónicas fluctúa entre 10 y $40 \%$ entre los 60 y 80 años, en cambio en nuestro estudio fue de $66,2 \%$; también señalan que las enfermedades prevalentes en esta etapa de la vida fueron: la hipertensión, enfermedades cardiovasculares, artritis y diabetes, que coindice con los resultados de nuestro estudio. Las enfermedades mentales también fueron más altas que en el estudio SABE ${ }^{(31)}$, en general, la prevalencia de enfermedades mentales en las PAMSC al ingreso al PNVD fue alrededor del $30 \%$, por ejemplo, en el estudio SABE, la prevalencia de depresión fluctuó entre 5 y $20 \%$, en nuestro estudio, fue del $100 \%$.
Nuestros hallazgos difieren con los encontrados en otros estudios realizados en PAMSC en otras latitudes como en Estados Unidos, Australia y Europa donde la depresión fluctúa entre 50 a $60 \%$ y la prevalencia de enfermedad mental se encuentra en $61 \%(4,32,33)$.

El estudio SABE también evaluó la dependencia funcional y se encontró que entre el 10 y $25 \%$ de las personas adultas mayores presentan, al menos, una limitación en las ABVD y AIVD (31). Nuestro estudio encontró que el $50 \%$ presenta limitaciones funcionales parciales. Además, la frecuencia de deterioro cognitivo fue cuatro veces mayor en las PAMSC que en los adultos mayores de la encuesta SABE. Nuestros resultados se alinean con los encontrado por otros estudios en la misma población en situación de calle ${ }^{(34)}$.

Otro hallazgo importante del presente estudio es que la limitación funcional parcial se relaciona directamente con la edad y el grado de deterioro cognitivo, como se observa en los investigaciones sobre envejecimiento ${ }^{(11,35)}$. Además, nuestro estudio muestra que la mujer es mucho más dependiente que el hombre, porque tiene mayor probabilidad de presentar limitación funcional, probablemente debido a que presenta mayor prevalencia de artritis y artrosis, osteoporosis, entre otras enfermedades ${ }^{(31)}$.

Este trabajo, a pesar de sus limitaciones por su diseño transversal y revisión de fichas de registro, brinda información valiosa no solo para el PNVD, sino también para la sociedad y el Estado peruano, porque hace la primera descripción sociodemográfica e identifica los problemas de salud física y mental de las PAMSC del Perú, que afortunadamente están siendo atendidos por el Estado a través del PNVD. Además, resalta que la limitación funcional y el deterioro cognitivo son factores importantes que considerar por el PNVD, porque de ello dependen los cuidados y servicios que se les brinde a este grupo vulnerable de personas, así como la distribución del presupuesto que ayuda a cubrir estas necesidades.

Si bien esta investigación provee evidencia sobre las PAMSC, es necesario realizar más investigaciones que permitan conocer a profundidad los motivos que hacen que estos peruanos terminen viviendo en la calle, además de generar iniciativas que evalúen el costobeneficio de los programas sociales que intervienen a poblaciones vulnerables como los adultos mayores en situación de calle.

Los resultados de esta investigación podrían servir para fortalecer la abogacía, la continuidad y la expansión de programas sociales, cuyo objetivo es devolverle los derechos y la ciudadanía a este grupo de peruanos altamente vulnerable. 
Contribuciones de autoría: MMR y CVM han participado en la concepción y redacción del artículo. MMR ha participado en la obtención del financiamiento. EL y MD han participado en la recolección de datos y revisión del artículo. CVM realizo el análisis de los datos.

Fuentes de financiamiento: este estudio ha sido financiado por el Programa Nacional Vida Digna (PNVD) del Ministerio de la Mujer y Poblaciones Vulnerables (MIMP).
Descargos de responsabilidad: los contenidos de este manuscrito solamente son responsabilidad de los autores y no necesariamente representan la opinión oficial del Programa Nacional Vida Digna (PNVD) del Ministerio de la Mujer y Poblaciones Vulnerables (MIMP).

Conflictos de interés: los autores reportan no tener conflictos de interés.

\section{REFERENCIAS BIBLIOGRÁFICAS}

1. Kelly PJ. Aging -- a public health success. Public Health Nurs. 2014 Jul-Aug;31(4):289. doi: 10.1111/ phn. 12132 .

2. Cohen CI, Sokolovsky J, Crane M. Aging, homelessness, and the law. Int J Law Psychiatry. 2001 MarJun;24(2-3):167-81.

3. Satariano WA, Guralnik JM, Jackson RJ, Marottoli RA, Phelan EA, Prohaska TR. Mobility and aging: new directions for public health action. Am J Public Health. 2012 Aug;102(8):1508-15. doi: 10.2105/AJPH.2011.300631.

4. Fischer PJ, Breakey WR. The epidemiology of alcohol, drug, and mental disorders among homeless persons. Am Psychol. 1991 Nov;46(11):1115-28.

5. Freeman RB, Hall B. Permanent homelessness in America?. Population Research and Policy Review. 1987;6(1):3-27.

6. Kondratas A. Ending homelessness. Policy challenges. Am Psychol. 1991 Nov;46(11):1226-31.

7. Instituto de Asistencia e Integración Social. Censo "Tú también cuentas IV”. 2011-2012. México: Instituto de Asisitencia e Integración Social; 2012.

8. Ministerio de Desarrollo Social. En Chile todos contamos. Segundo catastro nacional de personas en situación de calle. Chile: Ministerio de Desarrollo Social; 2011.

9. Ministerio de Planificación. Habitando la calle. Catastro Nacional de Personas en Situación de Calle, 2005. Chile: Ministerio de Planificación; 2005.

10. Centers for Disease Control (CDC). Deaths Among Homeless Persons -San Francisco, 1985-1990. MMWR Morb Mortal Wkly Rep. 1991 Dec 20;40(50):877-80.

11. Plumb JD. Homelessness: care, prevention, and public policy. Ann Intern Med. 1997 Jun 15;126(12):973-5.
12. Daiski I. Perspectives of homeless people on their health and health needs priorities. J Adv Nurs. 2007 May;58(3):273-81.

13. Dickey B. Review of programs for persons who are homeless and mentally ill. Harv Rev Psychiatry. 2000 Nov;8(5):242-50.

14. Tsai J, Mares AS, Rosenheck RA. Does housing chronically homeless adults lead to social integration?. Psychiatr Serv. 2012;63(5):427-34. doi: 10.1176/appi.ps.201100047.

15. de Vet $R$, van Luijtelaar MJ, Brilleslijper-Kater SN, Vanderplasschen W, Beijersbergen MD, Wolf JR. Effectiveness of case management for homeless persons: a systematic review. Am J Public Health. 2013 Oct;103(10):e13-26. doi: 10.2105/ AJPH.2013.301491.

16. Rodriguez RM, Fortman J, Chee C, Ng V, Poon D. Food, shelter and safety needs motivating homeless persons' visits to an urban emergency department. Ann Emerg Med. 2009 May;53(5):598-602. doi: 10.1016/j. annemergmed.2008.07.046.

17. Burt MR. Impact of housing and work supports on outcomes for chronically homeless adults with mental illness: LA's HOPE. Psychiatr Serv. 2012 Mar;63(3):209-15. doi: 10.1176/appi. ps.201100100.

18. Martinez TE, Burt MR. Impact of permanent supportive housing on the use of acute care health services by homeless adults. Psychiatr Serv. 2006 Jul;57(7):992-9.

19. Clark C, Rich AR. Outcomes of homeless adults with mental illness in a housing program and in case management only. Psychiatric Serv. 2003 Jan;54(1):78-83.

20. Kessell ER, Bhatia R, Bamberger JD, Kushel MB. Public health care utilization in a cohort of homeless adult applicants to a supportive housing program. J Urban Health. 2006 Sep;83(5):860-73.

21. Dorantes-Mendoza G, Ávila-Funes JA, Mejía-Arango S, GutiérrezRobledo LM. Factores asociados con la dependencia funcional en los adultos mayores: un analisis secundario del Estudio Nacional sobre Salud y Envejecimiento en Mexico, 2001. Rev Panam Salud Publica. 2007;22(1):1-11.

22. Perú, Congreso de la República. Programa Nacional Vida Digna. Decreto Supremo 006-2012-MIMP. Lima: Congreso de la República; 2012.

23. Trigás-Ferrín M, Ferreira-González L, Meijide-Míguez H. Escalas de valoración funcional en el anciano. Galicia Clin. 2012;72(1):11-6.

24. Organización Panamericana de la Salud. Evaluación funcional del adulto mayor. Washington D.C.: Organización Panamericana de la Salud.

25. Pfeiffer E. A short portable mental status questionnaire for the assessment of organic brain deficit in elderly patients. J Am Geriatr Soc. 1975 Oct;23(10):433-41.

26. Marc LG, Raue PJ, Bruce ML. Screening performance of the 15-item geriatric depression scale in a diverse elderly home care population. Am J Geriatr Psychiatry. 2008 Nov;16(11):914-21. doi: 10.1097/JGP.0b013e318186bd67.

27. Chongsuvivatwong V (2012). epicalc: Epidemiological Calculator. R package version 2.15.1.0.

28. Ruiz J, Aigneren M. Censo de adultos mayores en situación de calle, Medellín 2009. Medellín: Universidad de Antioquia, 2009.

29. Kondratas A. Estimates and public policy the politics of numbers. Housing Policy Debate. 1991;2(3):631-47. 
30. Chelimsky E. Politics, Policy Making, Data, and the Homeless. Housing Policy Debate. 1991;2(3):683-97.

31. Albala C, Lebrão ML, Leon Diaz EM, Ham-Chande R, Hennis AJ, Palloni A, et al. Encuesta Salud, Bienestar y Envejecimiento (SABE): metodología de la encuesta y perfil de la población estudiada. Rev Panam Salud Publica. 2005;17(5-6):307-22.

32. Rota-Bartelink A, Lipmann B. Causes of homelessness among older people in Melbourne, Australia. Aust N Z J Public Health. 2007 Jun;31(3):252-8.

33. Gaebel W, Zielasek J. Homeless and mentally ill - a mental healthcare challenge for Europe. Acta Psychiat Scand. 2015 Jan 24:236-8.

34. Cross W, Hayter M, Jackson D, Cleary M. Editorial: Meeting the health care needs associated with poverty, homelessness and social exclusion: the need for an interprofessional approach. J Clin Nurs. 2012 Apr;21(7-
8):907-8. doi: 10.1111/j.1365 2702.2011.03945.x.

35. National Institute on Aging. Biology of Aging. Research Today for a Healthier Tomorrow. Washington D.C: National Institute of Health; 2011.

Correspondencia: Marlene Moquillaza Risco Dirección: Jr. Camaná 616. Lima, Perú.

Correo electrónico:mmoquillaza@mimp.gob.pe

\section{http://twitter.com/rpmesp}

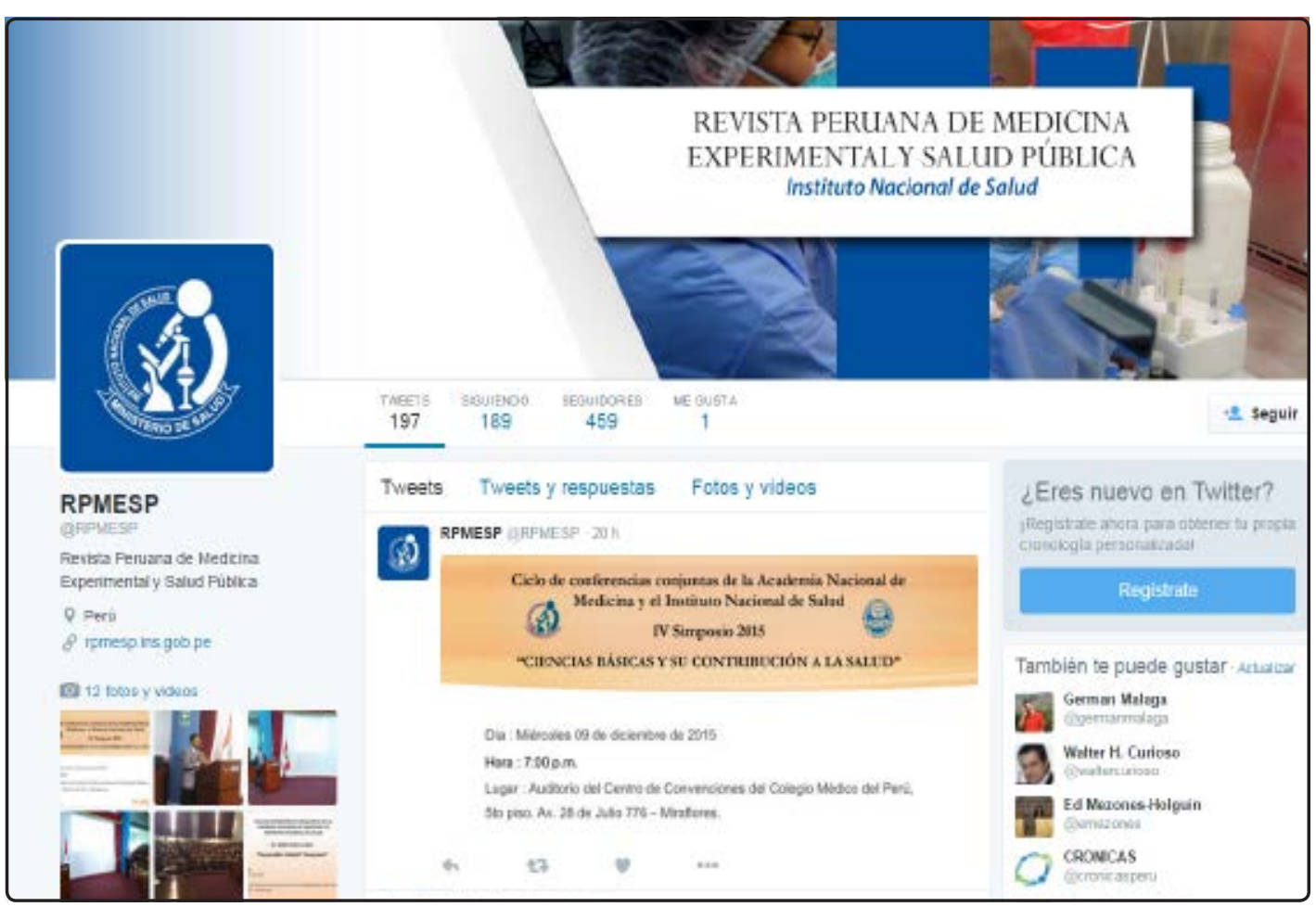

